\title{
NOTE ON OPERATIONS GENERATING THE GROUP OPERATIONS IN NILPOTENT GROUPS OF CLASS 3
}

\author{
ERNEST PLONKA \\ (Received 19 November 1973) \\ Communicated by G. E. Wall
}

Let $\boldsymbol{K}$ be a class of groups and let $\omega(\boldsymbol{K})$ denote the set of all such words $w(x, y)$ that the group operations $1, x^{-1}, x y$ in every group $G \in \boldsymbol{K}$ can be expressed as a superposition of $w$ and the projections $e_{1}(x, y)=x, e_{2}(x, y)=y$. Clearly,

$$
\omega(K) \supseteq\left\{x y^{-1}, x^{-1}, y, y x^{-1}, y^{-1} x\right\}
$$

for arbitrary class $\boldsymbol{K}$. The inverse does not hold in general (for example for a class of periodic nilpotent groups of class 2, see Hulanicki and Swierczkowski (1962), and one may ask about the class $\boldsymbol{K}$ for which

$$
\omega(K)=\left\{x y^{-1}, x^{-1} y, y x^{-1}, y^{-1} x\right) .
$$

Let $N_{k}$ be the variety of all nilpotent groups of class $k$. In Padmanabhan (1969) it is shown that $\left({ }^{*}\right)$ holds for the Abelian variety $N_{1}$, and recently Fajtlowicz (1972) has proved the same for $N_{2}$. One might conjecture that $\left(^{*}\right)$ is also valid for $N_{3}$. Unexpectedly enough, it turns out that this is not the case and in this note we prove the following result.

THEOREM. For all integers a we have

$$
x y^{-1}[y, x, x]^{a} \in \omega\left(N_{3}\right) .
$$

Proof. Let us recall the identities

$$
\begin{aligned}
& {[x y, z]=[x, z][x, z, y][y, z]} \\
& {[x, y z]=[x, z][x, y][x, y, z]}
\end{aligned}
$$

holding in any group. Using induction one can easily verify that the identities

$$
\begin{aligned}
& {\left[[x, y]^{m}, z\right]=[x, y, z]^{m}} \\
& {\left[x^{m}, y, z\right]=\left[x, y^{m}, z\right]=\left[x, y, z^{m}\right]=[x, y, z]^{m}} \\
& {\left[y^{n}, x^{m}\right]=[y, x]^{m n}[y, x, x]^{n\left(\frac{m}{2}\right)}[y, x, y]^{m\left(\frac{n}{2}\right)}}
\end{aligned}
$$


are fulfilled in every $G \in N_{3}$ and for all integers $m, n$. (For commutator identities see e.g. B. Huppert (1967)).

Let us observe that all operations of one variable can be expressed in terms of $w=x y^{-1}[y, x, x]^{a}$. Indeed, we have $w(x, x)=1, w(1, y)=y^{-1}$ and for all $k \geqq 0 x^{k+1}=w\left(x^{k}, x^{-1}\right) \cdot x^{-k-1}=w\left(x^{-k}, x\right)$. We verify that

$$
x y=w\left\{w\left(x, y^{-1}\right),\{w(w(w(y, w(y, x)), w(w(y, w(y, x)), x)), x)\} x^{-a}\right\} .
$$

Let

$$
\begin{aligned}
u & =w(y, w(y, x)) \\
& =w\left(y, y x^{-1}[x, y, y]^{a}\right) \\
& =y[x, y, y]^{-a} x y^{-1}\left[x^{-1}, y, y\right]^{a} \\
& =y x y^{-1}[y, x, y]^{2 a} \\
& =x[y, x][y, x, y]^{2 a-1}
\end{aligned}
$$

Hence

$$
\begin{aligned}
w(u, x) & =u x^{-1}[x, u, u]^{a} \\
& =x[y, x][y, x, y]^{2 a-1} x^{-1} \\
& =[y, x][y, x, x]^{-1}[y, x, y]^{2 a-1} .
\end{aligned}
$$

Then $v=w(u, w(u, x))=x[y, x, x]$, so that $w(v, x)=[y, x, x]$. But then we have $w\left(w\left(x, y^{-1}\right),(w(v, x))^{-a}\right)=x y$, which completes the proof of the theorem.

\section{References}

S. Fajtlowicz (1972), 'On fundamental operations in groups', J. Austral. Math. Soc. 14, 445-457.

A. Hulanicki and S. Swierzkowski (1962), 'On group operations other than $x y$ or $y x$ ', Publ. Math. Debrecen 9, 42-48.

B. Huppert (1967), Endliche Gruppen I, (Springer Verlag, 1967).

P. Padmanabhan (1969), 'A note on inverse binary operation in abelian groups' Fund. Math. 65, 61-63.

Instytut Matematyczny PAN

51-617 Wrocław

ul. Kopernika 18

Poland 